\title{
Does cognition influence the functional impairment degree of post-stroke patients?
}

Ozair Argentille Pereira da Silva', Gabriele Natane de Medeiros Cirne', Edson Meneses da Silva Filho', Enio Walker Azevedo Cacho', Roberta de Oliveira Cacho'

\begin{abstract}
Background: The post-stroke subjects have several sequelae after the disease, which should be investigated and identified so that the clinical treatment includes these people in all aspects. Objective: To identify if there is a correlation between level of education, cognitive function, functional independence and the sensory-motor abilities of post-stroke subjects. Methods: The sample consisted of 27 post-stroke patients who were evaluated through the following scales: Mini-Mental State Exam (MMSE), Functional Independence Measure (FIM) and Fugl-Meyer Assessment (FMAA) and sociodemographic data collection. Results: A positive and statistically significant correlation was found between MMSE and FIM. When the scores were correlated with education, motor FIM ( $p=0.005)$, total FIM ( $p=0.006)$, temporal orientation MMSE $(p=0.03)$ and total MMSE $(p=0.01)$ were statistically significant. Furthermore, a positive correlation but not statistically significant was identified between the cognitive levels and sensory-motor impairment assessed by FMAA. Conclusion: It is concluded that the education of subjects is associated with motor function after stroke. In addition, cognitive impairments interfere with the level of post-stroke functionality, but do not have a direct influence on the degree of sensory-motor impairment.
\end{abstract}

Keywords: Cognition; Neurology; Physiotherapy.

\section{INTRODUCTION}

Cerebrovascular accident or stroke is defined by the World Health Organization as a clinical sign of rapid focal disturbance of brain function, often of vascular origin and lasting for more than 24 hours $^{(1)}$. With regard to statistics, according to DATASUS, in the municipality of Santa Cruz located in the state of Rio Grande do Norte (Brazil), hospital admissions due to circulatory system disorders occurred in subjects older than 50 years had an incidence of more than $40 \%$ in $2009^{(2)}$. Another alarming data refers to the coefficient of mortality from cerebrovascular diseases that presented a prevalence of 66.2 per 100,000 inhabitants in 2008 . It is noteworthy that this rate is increasing every year since 2002 and currently occupies the second place of causes of death in this municipality ${ }^{(2)}$. Post-stroke patients often have several sequelae, such as changes in strength, coordination, and cognitive functions, which directly interfere with activities of daily living (ADL). It is emphasized that the early assessment of cognitive abilities is fundamental for the evolution of post-stroke patients ${ }^{(3)}$, because the cognitive ability of these subjects can be considered a prognostic factor for functional recovery ${ }^{(4)}$. The theory of the "Brain reserve" proposed by Katzman ${ }^{(5)}$, states that a high level of formal education increases the synaptic density of subjects in neocortical association areas, assuming that after a stroke these individuals could present less cognitive deficits. In contrast, the illiterates have the lowest corpus callosum causing levels of reduced cerebral connections between the cerebral hemispheres. Another factor that also directly interferes with post-stroke cognitive abilities, according to the National Institute of Health Stroke Scale (NIHSS) ${ }^{(6)}$, is the extent of the neurological damage ${ }^{(7)}$, which affects the higher cognitive functions and consequently the neurofunctional recovery ${ }^{(8)}$. Although there are several studies that have associated post-stroke injuries with cognitive problems ${ }^{(9,10)}$, there are still few associations between the cognitive aspects and the motor functions of these subjects, however it is believed that there is a positive correlation between them. In view of these assumptions, the present study aimed to analyze whether there is correlation between level of education, cognitive function, functional independence and the sensory-motor abilities of post-stroke patients.

\section{METHODS}

It is a cross-sectional study that followed the recommendations of the STrengthening the Reporting of OBservational studies in Epidemiology (STROBE)(11). It was performed in subjects 
with stroke sequelae, who were recruited by researchers and community health agents of the "Unidades Básicas de Saúde" (UBS's) of Santa Cruz (RN), Brazil. Subjects with clinical diagnosis of stroke, over 18 years old and with an injury time of more than 6 months were included in this study. The exclusion criteria were subjects who had others associated neurological pathologies, such as history of aneurysm, Parkinson's, Alzheimer's and subjects who suffered a new stroke event during the research. The study was approved by the Research Ethics Committee of the UFRN (protocol number 009/2011) and followed resolution of the National Health Council (no 196/96). The included patients were visited by the researchers, who were accompanied by the health agents and all were informed about the research procedures and signed the Informed Consent Form. Subsequently, the collection of socio-demographic data was performed through a semistructured questionnaire with the following information: name, gender, age, education, marital status, elapsed time since the first stroke. Soon after, the subjects were submitted to clinical and functional evaluation using the following measurement instruments: Mini-Mental State Exam (MMSE) to evaluate cognitive function ${ }^{(12)}$, Functional Independence Measure (FIM) ${ }^{(13)}$ to verify the level of functional independence and the Fugl-Meyer Assessment (FMAA) ${ }^{(14)}$ to evaluate the sensory-motor abilities.

The MMSE presents scores ranging from 0 to 30 points and has been validated in Brazil for Almeida ${ }^{(15)}$, who took into account the age and level of education to define the cut-off points. It is divided into seven dimensions, which include temporal orientation, spatial orientation, working memory, attention and calculation, recall, language and visual construction. The FIM evaluates the performance of the subject to perform a set of 18 tasks, referring to self-care, sphincter control, transfers, locomotion, communication and social cognition. Each item is classified according to the degree of dependence, with the value 1 corresponding to the total dependency and the value 7 corresponds to the accomplishment of tasks independently. Thus, the total score ranges from 18 to $126^{(13)}$. The FMA scale ${ }^{(14)}$ has a total of 226 points that are divided into 5 sub-items: motor function (100 points in total, 66 points for upper limb and 34 points for lower limb), sensory function (24 points), balance (14 points), range of motion (44 points) and joint pain ( 44 points). Each of these items are punctuated by an ordinal scale of 3 points ( 0 = cannot perform, 1 = partially performs, 2 = completely performs). In this study it was used only the score referring to motor function of upper limb and lower limb. The cutoff points used were as follows: up to 35 points characterized as severe motor impairment; between $36-55$ points characterized as severely moderate motor impairment; between $56-79$ points characterized as moderate motor impairment; and 80 points or more characterized as mild motor impairment ${ }^{(16)}$. The Shapiro-Wilk test was used to verify the normality of the data and also the descriptive analysis was done. The Pearson correlation test was used to calculate the correlation between level of education, cognitive functions and sensory-motor abilities. The comparisons between the illiterate and literate groups were made by the t-test for 2 samples. It was used a level of significance of $5 \%$.

\section{RESULTS}

Initially the sample consisted of 42 subjects with stroke sequels. Of them, 15 were excluded: 11 had a stroke in less than 6 months, 3 had another associated neurological pathology, and 1 had a new stroke event during the study. Thus, 27 subjects participated in the study. All sample characterization is shown in Table 1.

The results of the correlation between the total MMSE and the motor FIM scores showed a positive and statistically significant correlation, as well as between the total MMSE and FIM, demonstrating that the higher the level of cognition presented by the subjects the better the degree of functional independence (Table 2). Furthermore, a positive correlation but not statistically significant was identified between the total MMSE and the FMAA (upper limb, lower limb and both) (Table 3).

Table 1. Socio-demographic characterization of the sample.

\begin{tabular}{|c|c|}
\hline Variables & Patients $(n=27)$ \\
\hline \multicolumn{2}{|l|}{ Gender (\%) } \\
\hline Male & 55 \\
\hline Female & 45 \\
\hline Age (years) & $67.15 / 14.77$ \\
\hline \multicolumn{2}{|l|}{ Marital status (\%) } \\
\hline Married & 66.7 \\
\hline Widower/Widow & 22.2 \\
\hline Single & 11.1 \\
\hline \multicolumn{2}{|l|}{ Level of Education } \\
\hline Literate & 48 \\
\hline Illiterate & 52 \\
\hline \multicolumn{2}{|l|}{ Type of injury (\%) } \\
\hline Ischemic & 63 \\
\hline Hemorrhagic & 26 \\
\hline Not specified & 11 \\
\hline \multicolumn{2}{|l|}{$\mathrm{N}^{\circ}$ of events $(\%)$} \\
\hline One event & 67 \\
\hline Two event & 22 \\
\hline Three event & 11 \\
\hline FMAA total (Mean/SD) & $137.47 / 54.35$ \\
\hline FIM total (Mean/SD) & $80.18 / 31.67$ \\
\hline MMSE total (Mean/SD) & $17.74 / 6.68$ \\
\hline
\end{tabular}

Note: FMAA: Fugl-Meyer Assessment; MMSE: Mini-Mental State Exam; FIM: Functional Independence Measure; SD: Standard Deviation. 
To verify the correlations between level of education, functional independence and cognitive levels, the education of the patients was correlated with the FIM and MMSE scores. The MMSE was sub-divided into 6 domains (1: temporal orientation; 2: spatial orientation; 3: Working memory; 4: Attention and calculation; 5: Recall; 6: Language and writing), and the FIM was divided in 2 domains (cognitive and motor). Table 4 shows that, when correlated with education, the motor FIM and total FIM scores were statistically significant, demonstrating that the literate population has lower functional impairments than the illiterate.

Table 2. Correlation between the Mini-Mental State Exam and the Functional Independence Measure.

\begin{tabular}{lcc}
\hline \multicolumn{1}{c}{ Correlation } & $\mathbf{r}$ & p-value \\
\hline total MMSE X motor FIM & 0.4943 & $0.008^{*}$ \\
total MMSE X total FIM & 0.5898 & $0.001^{*}$ \\
\hline
\end{tabular}

Note: MMSE: Mini-Mental State Exam; FIM: Functional Independence Measure r: Pearson correlation; ${ }^{*}$ p-value statistically significant.

Table 3. Correlation between the Mini-Mental State Exam and the Fugl-Meyer Assessment.

\begin{tabular}{lcl}
\hline \multicolumn{1}{c}{ Correlation } & $\boldsymbol{r}$ & p-valor \\
\hline total MMSE X UL-FMAA & 0.2899 & 0.1797 \\
total MMSE X LL-FMAA & 0.3423 & 0.1099 \\
total MMSE X total FMAA & 0.3809 & 0.073
\end{tabular}

Note: MMSE: Mini-Mental State Exam; FMAA: Fugl-Meyer Assessment; UL: Upper limb; LL: Lower limb; r: Pearson correlation.

Table 4. Influence of education on Functional Independence Measure.

\begin{tabular}{|c|c|c|c|}
\hline \multirow{2}{*}{ Variables } & Illiterate & Literate & \multirow{2}{*}{$p$-value } \\
\hline & Mean (SD) & Mean (SD) & \\
\hline motor FIM & $41.53(22.46)$ & $67.85(22.47)$ & $0.005^{*}$ \\
\hline cognitive FIM & $21.76(8.90)$ & $28.00(8.66)$ & 0.06 \\
\hline Total FIM & $63.30(26.66)$ & 95.85 (28.29) & $0.006^{*}$ \\
\hline MMSE 1 & 2.69 (2.09) & $4.28(1.32)$ & $0.03 *$ \\
\hline MMSE 2 & 3.76 (1.64) & $4.42(1.34)$ & 0.28 \\
\hline MMSE 3 & $2.46(1.12)$ & 2.35 (1.27) & 1.00 \\
\hline MMSE 4 & 0.615 (1.55) & $0.92(1.45)$ & 0.21 \\
\hline MMSE 5 & $0.923(1.11)$ & $1.5(1.34)$ & 0.28 \\
\hline MMSE 6 & $5.15(2.07)$ & $6.42(2.34)$ & 0.06 \\
\hline total MMSE & $15.38(6.04)$ & 19.92 (6.69) & $0.01 *$ \\
\hline
\end{tabular}

Note: MMSE: Mini-Mental State Exam; FIM: Functional Independence Measure; $r$ : Pearson correlation; *p-value statistically significant. MMSE 1: temporal orientation MMSE 2: spatial orientation; MMSE 3: Working memory; MMSE 4: Attention and calculation; MMSE 5: Recall; MMSE 6: Language and writing; total MMSE: total value; SD: Standard deviation.

\section{DISCUSSION}

This study demonstrated that post-stroke literate subjects had higher cognitive and motor abilities than those who were illiterate. Corroborating with these results, Costa et al. ${ }^{(17)}$ identified that literate subjects presented favorable evolution in the first 6 months after stroke and this is also related to their clinical improvement. Early assessments of post-stroke cognitive aspects allow the therapist to identify the patient's potential for rehabilitation to make concrete treatment plans ${ }^{(3)}$. It is important to emphasize that after brain injury, in addition to motor disorders, some patients may develop difficulties in the use of the already acquired cognitive ability, as well as in the acquisition of new knowledge. The dysfunctions that can occur in memory, attention, logical reasoning and reading are pointed out as the most common cognitive deficits. These changes may be present depending on the lesion location and severity and, therefore, vary significantly between subjects ${ }^{(18)}$. Another study that is in agreement with our results showed that patients without previous neurological diseases and those who had the first stroke presented differences in cognitive deficits according to educational levels ${ }^{(19)}$. Cognitive skills, besides being fundamental for the patient to understand their physical impairment, also affect the ability to learn and perform functional tasks ${ }^{(3)}$. In the present study, a high percentage of stroke subjects with low levels of education were observed, drawing attention to the number of illiterates (52\%). The low amount of information about the means of prevention, the habits and behaviors of health risk make the population of low education more prone to stroke ${ }^{(20)}$. Functional independence requires greater cognitive abilities to perform, for example, activities of daily living, moreover, requires the interpretation and solution of problems that are highly dependent on cognitive levels. On the other hand, since the motor evaluation by FMA does not depend on problem solving, this may be an explanation for the non-correlation with cognitive damage ${ }^{(21)}$.

This study presented as limitations the low number of patients included in the sample who may have limited the identification of differences between some variables between the groups. In addition, the generalization of these results should be done with caution. Finally, it is emphasized that the evaluation instruments used, although simple, are validated and relevant due to their great clinical applicability.

\section{CONCLUSION}

The present study showed that cognitive function was positively correlated with the motor functionality, i.e., the higher the cognitive levels the more independent the subject. Cognitive function also showed a positive correlation with the degree of sensory-motor impairment, but the results were not significant, which does not allow any existing relationship to be established. 


\section{AUTHORS' CONTRIBUTIONS}

EWAC and ROC: study design. OAPS, GNMC and EMSF: data collection. ROC: intellectual critical review of the manuscript. All authors read and approved the final version of the manuscript.

\section{CONFLICT OF INTEREST}

The authors declare that there was no conflict of interest.

\section{REFERENCES}

1. Organização Mundial da Saúde. Health topics Stroke, Cerebrovascular accident. 2018. Disponível em http://www.who.int/topics/ cerebrovascular_accident/en/.

2. Ministério da Saúde. Datasus. Mortalidade e Morbidade Hospitalar: Santa Cruz - Rio Grande do Norte. Disponível em: www.datasus.gov.br.

3. Zwecker M, Levenkrohn S, Fleisig Y, Zeilig G, Ohry A, Adunsky A. MiniMental State Examination, cognitive FIM instrument, and the Loewenstein Occupational Therapy Cognitive Assessment: relation to functional outcome of stroke patients. Arch Phys Med Rehabil. 2002; 83(3): 342-5.

4. Park J, Lee SU, Jung SH. Prediction of post-stroke functional mobility from the initial assessment of cognitive function. NeuroRehabilitation. 2017; 41(1): 169-177.

5. Katzman R. Education and the prevalence of dementia and Alzheimer's disease. Neurology. 1993; 43: 13-20.

6. National Institute of Health Stroke Scale (NIHSS). Disponível em https:// www.mdcalc.com/nih-stroke-scale-score-nihss.

7. Costa FA, Silva DLA, Rocha VM. Estado neurológico e cognição de pacientes pós-acidente vascular cerebral. Rev Esc Enferm USP. 2011; 45(5): 1083-8.

8. Zinn S, Dudley TK, Bosworth HB, Hoenig HM, Duncan PW, Horner RD. The effect of poststroke cognitive impairment on rehabilitation process and functional outcome. Arch Phys Med Rehabil. 2004; 5: 1084-90.

9. Lo Coco D, Lopez G, Corrao S. Cognitive impairment and stroke in elderly patients. Vasc Health Risk Manag. 2016; 12: 105-16.
10. Merriman NA, Sexton E, Donnelly NA, McCabe G, Walsh ME, Rohde $D$, et al. Managing cognitive impairment following stroke: protocol for a systematic review of non-randomised controlled studies of psychological interventions. BMJ Open. 2018; 8(1): e019001.

11. Von Elm E, Altman DG, Egger M, Pocock SJ, Gøtzsche PC, Vandenbroucke $J P$, et al. The Strengthening the Reporting of Observational Studies in Epidemiology (STROBE) Statement: guidelines for reporting observational studies. Int J Surg. 2014; 12(12): 1495-9.

12. Folstein MF, Folstein SE, McHugh PR. Mini-mental state: a practical method for grading the cognitive state of patients for clinician. J Psychiatc Res. 1975; 12: 189-198.

13. Riberto M, Miyazaki MH, Jucá SSH, Sakamoto H, Pinto PPN, Battistella LR. Validação da Versão Brasileira da Medida de Independência Funcional. Acta Fisiatr. 2004; 11(2): 72-6.

14. Fugl-Meyer AR, Jaasko L, Leyman L, Olsson S, Steglind S. The post-stroke hemiparetic patients. A method for evaluation of physical performance. Scand J Rehab Med. 1975; 7(1): 13-31.

15. Almeida OP. Mini Exame do Estado Mental e o diagnóstico de demência no Brasil. Arq Neuropsiquiatr. 1998; 56(3B), 605-12.

16. Cacho EWA, Melo FRLV, Oliveira R. Avaliação da recuperação motora de pacientes hemiplégicos através do protocolo de desempenho físico FuglMeyer. Rev Neurociências. 2004; 12(2): 94-102.

17. Costa FA, Bezerra IFD, Silva DLA, Oliveira R, Rocha VM. Cognitive evolution by MMSE in post stroke patients. Int J Rehabil Res. 2010; 33(3): 248-53.

18. Osmon DC, Smet IC, Winegarden B, Gandhavadi B. Neurobehavioral Cognitive Status Examination: its use with unilateral stroke patients in a rehabilitation setting. Arch Phys Med Rehabil. 1992; 73: 414-8.

19. Rasquin SMC, Verhey FRJ, Van Oostenbrugge RJ, Lousberg R, Lodder J. Demographic and CT scan features related to cognitive impairment in the first year after stroke. J Neurol Neurosurg Psychiatry. 2004; 75: 1562-67.

20. Huang ZS, Chiang TL, Lee TK. Stroke prevalence in Taiwan: findings from the 1994 National Health Interview Survey. Stroke. 1997; 28: 1579-84.

21. Rajji TK, Miranda D, Mulsant BH. Cognition, Function, and Disability in patients with schizophrenia: a review of longitudinal studies. Can J Psychiatry. 2014; 59(1): 13-7. 